\title{
Impacto do Método Pilates na qualidade de vida dos praticantes
}

\author{
The impact of Pilates Method on quality of life of practitioners
}

Josiane Teresinha Bertoldi', Rafael Tesser', Mayara dos Santos Damaceno'

${ }^{1}$ Faculdade Guilherme Guimbala - Associação Catarinense de Ensino (FGG/ACE), Joinville, 5C, Brasil.

Recebido em: 31/03/2016 / Aceito em: 11/04/2016

rafaeltesser@hotmail.com

\section{RESUMO}

Objetivo: avaliar o impacto do Método Pilates (MP) na qualidade de vida ( $\mathrm{QV}$ ) de seus praticantes, após três meses de atividade. Método: o estudo foi realizado por meio de um protocolo geral de identificação, anamnese e o questionário Medical Outcome Study Short-Form 36 Health Survey (SF-36) para QV. Os critérios de inclusão englobaram iniciantes no MP, excluindo aqueles que praticavam há mais de um mês, que realizassem outro exercício físico concomitante, em situação de pós-operatório e/ou infecções ativas. A amostra foi composta por 23 indivíduos, sendo 16 mulheres e 07 homens, idade média 40,17 $( \pm 18,09)$ anos. Resultados: apontaram melhora da QV na faixa etária até 30 anos (2\%); de 41 a 50 anos $(8 \%)(p<0,05)$ e acima de 61 anos $(2 \%)$. Entre os sexos, houve ganho na QV do sexo feminino (4\%) $(p<0,05)$. Em relação à frequência semanal, o aumento da QV foi identificado para $02 x /$ sem (3\%) e $03 x /$ sem $(6 \%)(p<0,05)$. Comparando o resultado entre o grupo saudável (GS) e o grupo com patologias (GP), notou-se escore inalterado para GS e acréscimo na QV para o GP $(4 \%)(p<0,05)$. Considerações finais: após três meses de prática, o MP, promoveu discreta evolução na QV dos praticantes, com destaque para a faixa etária entre 41 e 50 anos, para o sexo feminino, para a maior frequência semanal e para pacientes com patologias.

Palavras-chave: Pilates; Qualidade de Vida; Atividade Física.

\section{ABSTRACT}

Objective: of this research was to evaluate the impact of the Pilates Method (PM) on quality of life (QOL) of practitioners, after three months. Method: the study was conducted through a general protocol of identification, history and Medical survey Outcome Study Short-Form 36 Health Survey (SF-36) to QOL. The inclusion criteria were beginners in the $P M$, excluding those who practiced for over a month, which perform other concomitant physical exercise in post-operative situation and/or active infections. The sample was composed of 23 individuals, being 16 women and 07 men, average age 40.17 ( \pm 18.09) years. Results: showed improvement of $Q O L$ in the age group up to 30 years $(2 \%)$ of the $41-50$ years $(8 \%)(p<0,05)$ and over 61 years (2\%). Between the sexes, there was gain in women $Q O L(4 \%)(p<0,05)$. In relation to weekly frequency increased $\mathrm{OOL}$ was identified for $02 \mathrm{x} / \mathrm{sem}$ $(03 x / 3 \%)$ and without $(6 \%)(p<0,05)$. Comparing the results between the healthy Group (HG) and the group with pathologies (GP), observed score unchanged for $H G$ and increase in $Q O L$ for GP (4\%) $(p<0,05)$. Closing remarks: after three months of practice, the $P M$, promoted developments in discrete Qol of practitioners, especially for the age group between 41 and 50 years for females, for the biggest weekly frequency, and for patients with pathologies.

Keywords: Pilates Method; Quality of Life; Physical Activity.

\section{INTRODUCÃ̃}

A qualidade de vida (OV) é definida pela Organização Mundial da Saúde (OMS), como a percepção do indivíduo sobre a sua posição na vida, no contexto da cultura e dos sistemas de valores nos quais ele vive e em relação aos seus objetivos, expectativas, padrões e preocupações. ${ }^{1}$ A QV pode ser delimitada em: não relacionada à saúde e relacionada à saúde (OVRS). ${ }^{2}$ Esta última coloca sua centralidade na capacidade de viver sem doenças ou de superar as dificuldades dos estados 
ou condições de morbidade. ${ }^{3}$

O sedentarismo e o estresse são fatores responsáveis por doenças hipocinéticas, enquanto que a prática regular de atividade física está associada a menor mortalidade e consequentemente melhor percepção da QVRS. ${ }^{2,4} \mathrm{O}$ estudo de Gordia et al. ${ }^{1}$ indica que independente de idade, raça, sexo, nível educacional, tabagismo e o índice de massa corporal (IMC), indivíduos que praticam atividade física em níveis recomendados apresentam maior chance de possuir QV boa, quando comparados com seus pares inativos. ${ }^{1} \mathrm{~A}$ prática de exercícios regulares, além de promover a saúde, influencia na reabilitação de determinadas patologias associadas ao aumento dos índices de morbidade e da mortalidade. ${ }^{5}$ Com isso, o desenvolvimento de ações preventivas e de reabilitação tem sido objeto de trabalho de profissionais da saúde, principalmente da fisioterapia. ${ }^{1,4}$

Dentre as opções, destaca-se o método Pilates, que atua promovendo a saúde integral, engajamento total do corpo e a respiração. ${ }^{6}$ Esta técnica de condicionamento consiste em exercícios físicos, cuja característica principal é o trabalho resistido e o alongamento dinâmico. Os benefícios da prática englobam: coordenação, força mobilidade, eficiência do movimento, fluidez do movimento, postura adequada, restauração do movimento animal natural, integração da mente, corpo e espírito, sensação de bem-estar e melhor QV. ${ }^{4,7,8}$

Desta forma, o objetivo deste estudo foi avaliar o impacto da prática regular de Pilates, na percepção da qualidade de vida e seus aspectos, após três meses de atividade.

\section{MÉTODO}

A pesquisa foi aprovada pelo Comitê de Ética em pesquisa, CEP/IELUSC, Joinville-SC, por meio do parecer $n^{\circ} 992.004$ de 19/03/2015. Trata-se de uma pesquisa do tipo descritiva com abordagem quantitativa e experimental.

O estudo iniciou com a assinatura do Termo de Consentimento Livre e Esclarecido, em conformidade com a Resolução 466/2012 CNS/CONEP pelos pacientes. O público-alvo da pesquisa foi constituído de iniciantes no método Pilates, que nunca praticaram ou com no máximo um mês nesta atividade, além de pacientes que estivessem retornando, porém estiveram mais de um mês afastados. Os critérios de exclusão abrangiam pacientes de Pilates há mais de um mês ou que praticavam outra atividade concomitantemente, pacientes em pós-operatórios e infecções ativas. Isto posto, a amostra inicial foi composta por 39 pacientes, selecionados em estúdios de Pilates parceiros desta pesquisa, localizados em Joinville e região: Equilibrium Pilates Estúdio, Estúdio Corefit, Pilates One, Rtesser Pilates, Wellness Estúdio, e Studio de Pilates Fisio.Ru.

As avaliações incluíram um protocolo geral e um protocolo específico. O protocolo geral foi composto por uma ficha para preenchimento dos dados de identificação, a anamnese englobando o histórico pregresso/ atual e familiar, bem como questões acerca da prática de atividade física, tempo de prática e quais modalidades praticadas. No específico, para avaliar a QV foi utilizado o Medical Outcome Study Short-Form $36 \mathrm{He}$ alth Survey (SF-36), criado por Ware e Sherbourne em
$1992,{ }^{9}$ originalmente na língua inglesa norte-americana. No Brasil, teve sua tradução e validação cultural realizada por Ciconelli et al. ${ }^{10}$ Este instrumento é composto por 36 questões, abrangendo oito domínios relacionados à saúde física e mental na percepção das últimas quatro semanas vividas pelo avaliado. Os domínios que correspondem à saúde física são: funcionalidade física, limitações físicas, dor corporal e saúde geral. Os que correspondem à saúde mental são: vitalidade, relações sociais, limitações emocionais e saúde mental. Cada domínio é quantificado por uma escala de 0 a 100, sendo zero, o pior esultado, e 100 o melhor resultado em QV. ${ }^{1}$

Após três meses, realizou-se a avaliação final com os mesmos protocolos utilizados inicialmente. Cabe ressaltar que cada sessão durou em média 50 minutos. Os protocolos de exercícios aplicados foram definidos pelos instrutores responsáveis, conforme condição e capacidade dos clientes no momento da avaliação postural e física própria de cada estúdio. A frequência semanal também variou entre uma, duas ou três vezes por semana, de acordo com a possibilidade de cada cliente. Da amostra inicial, houve 13 desistências da prática, três questionários não respondidos corretamente ou no prazo estipulado; finalizando a pesquisa com 23 participantes efetivos, sendo 16 do sexo feminino e sete do sexo masculino, com média de idade de 40,17 anos $( \pm 18,09)$.

Os dados coletados foram avaliados pelo comparativo inicial e final, calculados os domínios do questionário utilizado de acordo com o proposto na literatura, posteriormente por meio de planilhas do Microsoft Office Excel ${ }^{\circledR}$ 2013, com cálculo da média, desvio-padrão e percentual, separando por fatores que podem influenciar na percepção da QV: idade, sexo, frequência semanal e presença de patologias ou não. Para comparar as pontuações do SF-36 em cada domínio, obtidas nas avaliações inicial e final, foi utilizado o teste $\mathrm{t}$, para amostras pareadas. O nível de significância foi estabelecido em $\mathrm{p}<0,05$.

\section{RESULTADOS}

Na avaliação da QV, por meio do questionário SF36, observou-se um acréscimo total após três meses. No que se refere à QVRS, os domínios "capacidade funcional", "dor" e "estado de saúde geral" apresentaram evolução discreta entre o pré-teste e o pós-teste. No que tange à $\mathrm{QV}$ relacionada à saúde mental, verificou-se que os domínios "vitalidade", "aspectos sociais" e "saúde mental" obtiveram melhora, sendo significativas nos dois primeiros $(p<0,05)$, conforme exposto na tabela 1 .

Em relação aos resultados por faixa etária, expostos no gráfico 1, houve ganhos em todos os subgrupos: $2 \%$ na QV do subgrupo até 30 anos, $8 \%$ para o subgrupo de 41 a 50 anos $(p<0,05)$ e $2 \%$ para os maiores de 61 anos. Na faixa etária de 31 a 40 anos e entre 51 e 60 anos, não houve alteração entre pré e pós-teste.

No gráfico $n^{\circ} 2$ está demonstrada a classificação por sexo, apontando um ganho na QV para o sexo feminino de $4 \%(p<0,05)$, enquanto que o público masculino não apresentou variação significativa.

No tocante à frequência, aqueles que executaram o método apenas uma vez durante a semana obtiveram 
IMPACTO DO MÉTODO PILATES NA QUALIDADE DE VIDA DOS PRATICANTES

Josiane Teresinha Bertoldi, Rafael Tesser, Moyara dos Santos Damaceno.

Tabela 1 - QV (SF-36) média geral dos escores por domínios, após 03 meses de MP.

\begin{tabular}{|c|c|c|c|c|}
\hline & SF 36 & PRÉ & PÓS & RESULTADO \\
\hline \multirow[t]{3}{*}{ SAÚDE FÍSICA } & Capacidade Funcional & 82,17 & 84,35 & $2 \%$ \\
\hline & Dor & 66,22 & 69,35 & $3 \%$ \\
\hline & Estado de Saúde Geral & 76,83 & 78,48 & $2 \%$ \\
\hline \multirow[t]{4}{*}{ SAÚDE MENTAL } & Vitalidade & 65,87 & 69,35 & $4 \% *$ \\
\hline & Aspectos Sociais & 77,72 & 83,70 & $6 \% *$ \\
\hline & Saúde Mental & 76,17 & 76,35 & $1 \%$ \\
\hline & Escore total: & 606,95 & 619,20 & $2 \%$ \\
\hline
\end{tabular}

${ }^{*}$ estatisticamente significativos $p<0,05$

Gráfico 1 - Escore total QV (SF-36) classificação por faixa etária.

\section{QUALIDADE DE VIDA}

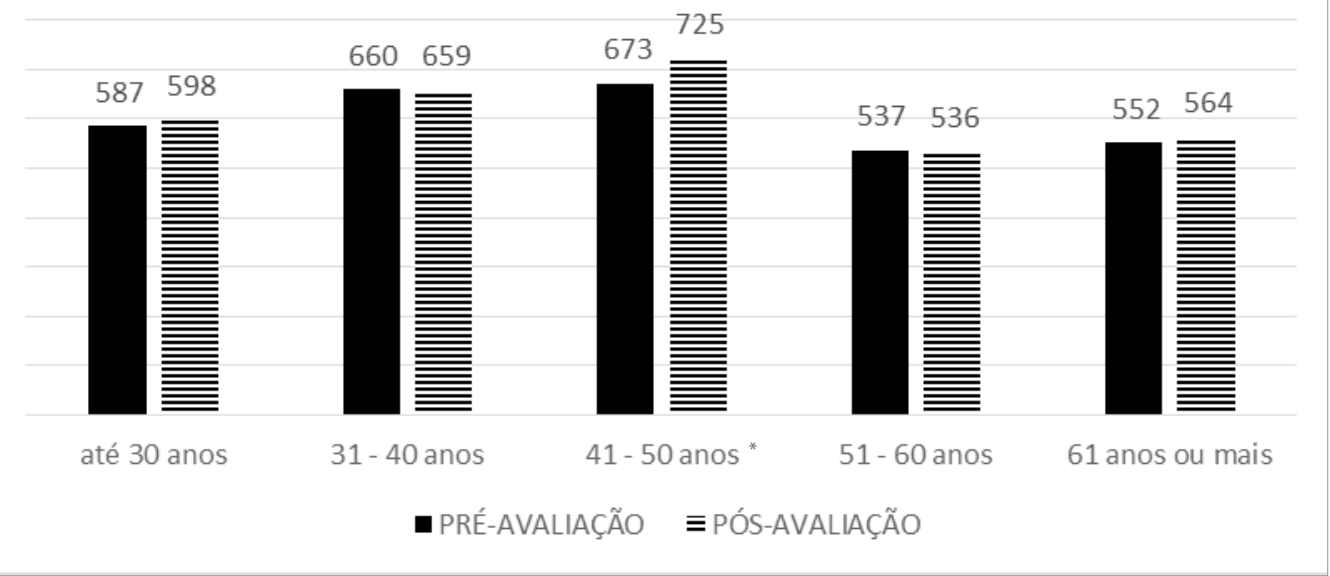

*estatisticamente significativo $p<0,05$

Gráfico 2 - Escore total QV (SF-36) classificação por gênero.

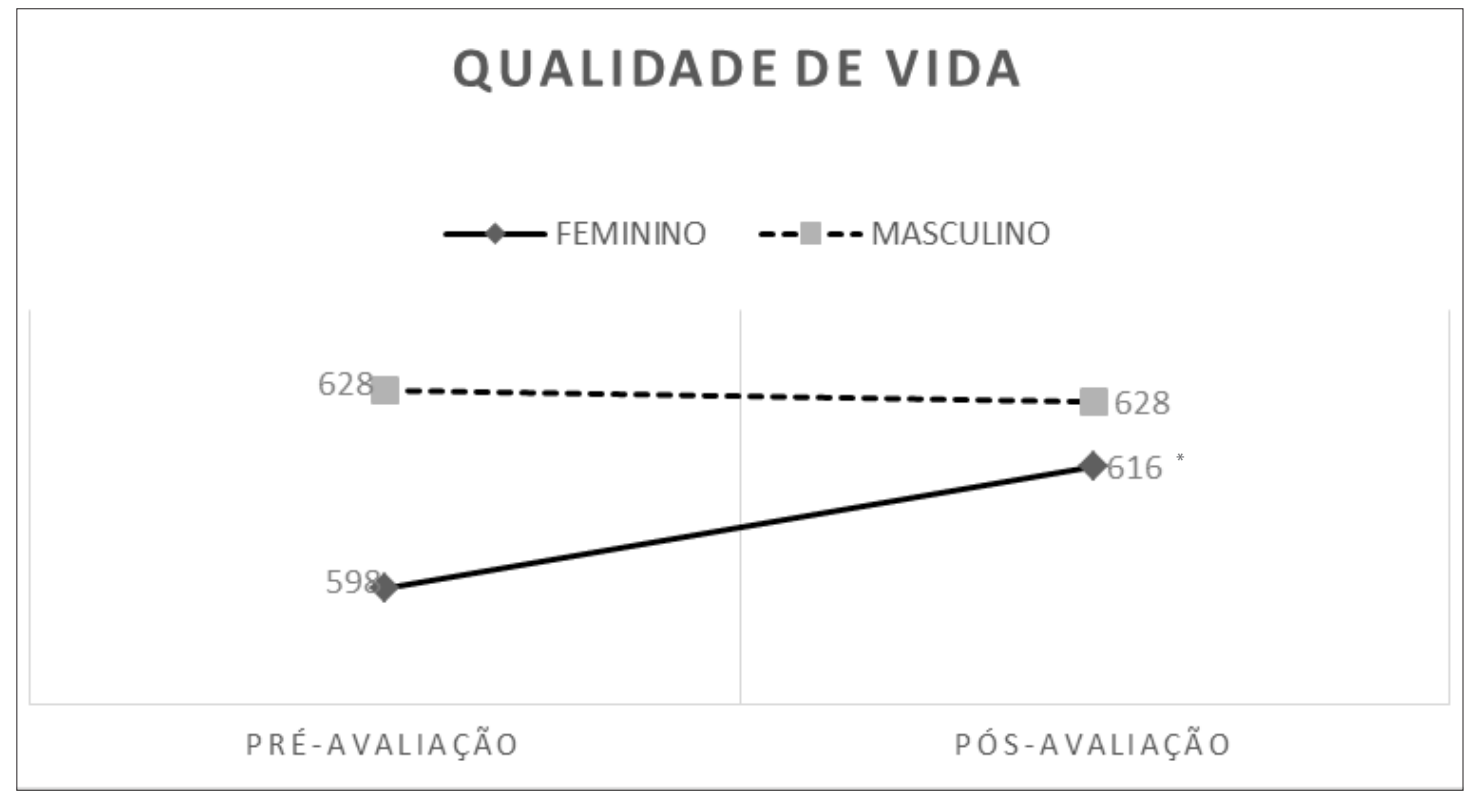


Gráfico 3 - Escore total QV (SF36) classificação por frequência semanal.

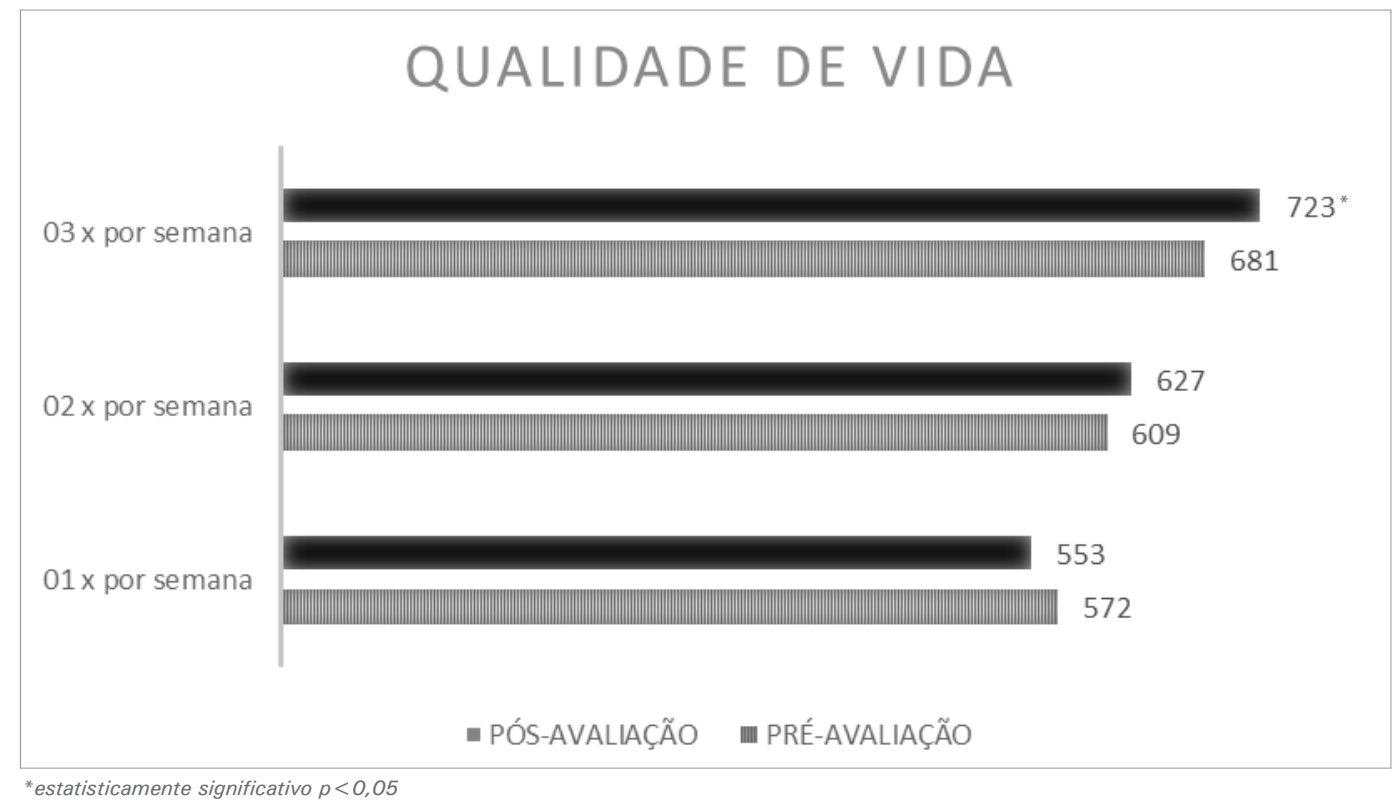

redução de $3 \%$ na pontuação final, ao contrário daqueles que se exercitavam com maior frequência, constatando um aumento de $3 \%$ no escore final para duas vezes na semana, e um acréscimo significativo de $6 \%(p<0,05)$ na QV para aqueles que frequentavam três dias na semana, conforme exibido no gráfico $\mathrm{n}^{\circ} 3$.

No comparativo geral da QV, através do histórico colhido inicialmente, foi possível classificar os participantes em dois grupos: sem nenhuma patologia diagnosticada, o qual denominou-se grupo saudável (GS) e o grupo com patologia diagnosticada (GP). Constatou-se que o segundo evoluiu em $4 \%(p<0,05)$, após três meses, enquanto que o primeiro não sofreu alteração no escore. Destaca-se que a OV para o primeiro grupo já era maior antes do início da prática.

Contemplando o perfil da amostra GP, observou-se que as patologias são de origens diversas: músculo-esqueléticas, gastrointestinais, uroginecológicas, vascu- lares, neurológicas e ópticas. Assim sendo, foi possível observar a análise por tipo de patologia, sendo os maiores escores percebidos para enxaqueca e lombalgia, ampliando em $20 \%$ e $17 \%$ respectivamente, a OV destes pacientes, conforme gráfico 4 .

\section{DISCUSSÃO}

A qualidade de vida (OV) é uma noção eminentemente humana que se aproxima do grau de satisfação encontrado na vida familiar, amorosa, social e ambiental. Pressupõe uma síntese cultural de todos os elementos que determinada sociedade considera como seu padrão de conforto e bem-estar. ${ }^{11}$

No que concerne à saúde, o exercício físico surge como um dos meios para aprimorar o conceito no que se refere ao bem-estar físico, social e emocional. ${ }^{12} \mathrm{~A}$

Gráfico 4 - Resultado da evolução da QV (SF-36) por patologias.

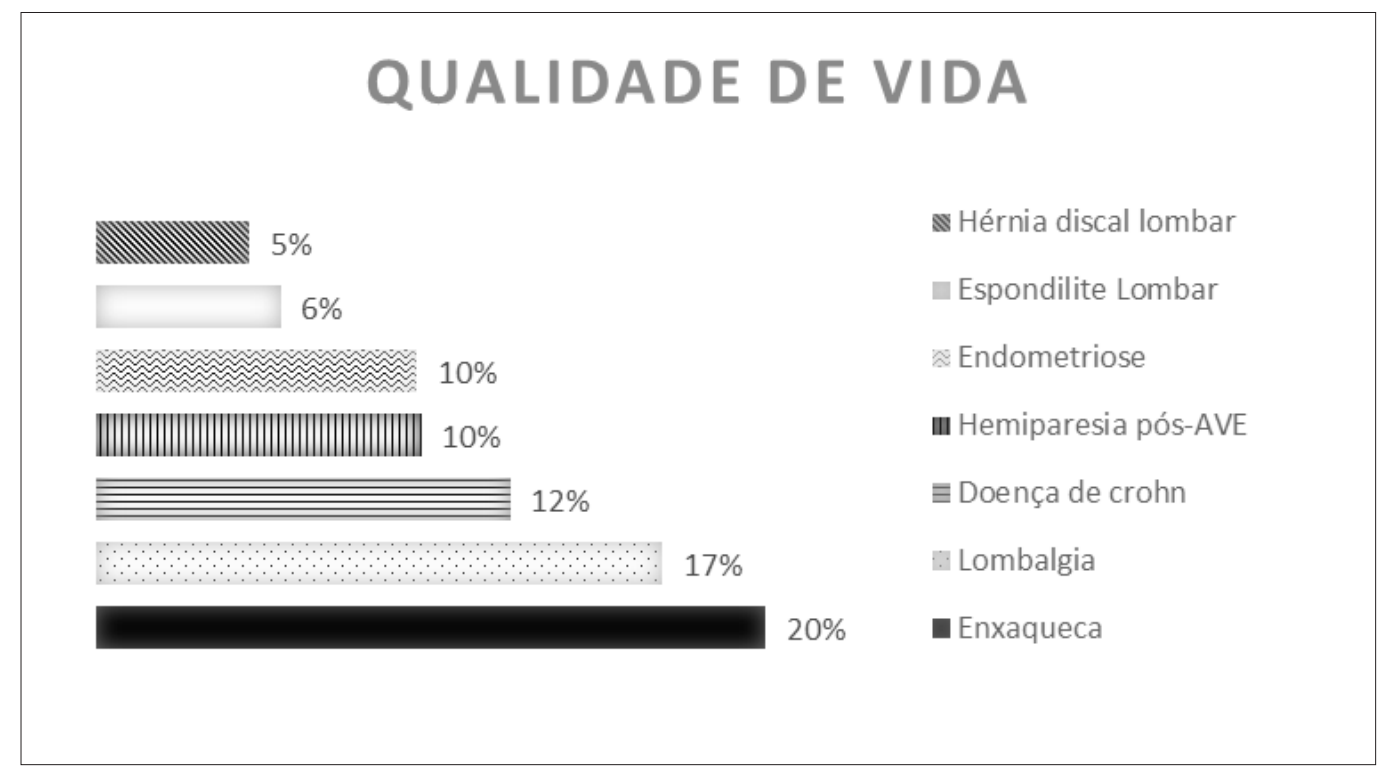


QV está diretamente relacionada à aptidão física e à saúde $^{2}$. Assim, observa-se que o MP como modalidade de exercício físico, dependendo do tipo, intensidade, duração e frequência, ocasiona diversas alterações hormonais acompanhado de mudanças bioquímicas e fisiológicas. ${ }^{13}$ Está associado à melhor qualidade dos valores da vida, sobretudo nas dimensões do funcionamento físico, saúde geral e saúde mental. ${ }^{2}$

Para Takata et al., ${ }^{14}$ que avaliaram a associação da QV pelo SF-36 e aptidão física em 207 participantes, com média de idade de 85 anos, sugerem que a melhora do nível de aptidão física, mesmo no envelhecimento, pode aumentar a QV. Outro estudo mostrou que idosos praticantes do método Pilates tem uma melhor $\mathrm{QV}$, quando comparado com idosos que não praticavam o método. ${ }^{15}$ Pesquisas apresentam o Pilates como um método alternativo e eficaz para melhorar a QV em idosos, adultos jovens saudáveis, entre outros. ${ }^{16}$ Esta pesquisa obteve resultados positivos em diferentes faixas etárias, demonstrando que as amostras: abaixo de 30 anos, entre 41 e 50 anos e também acima de 60 anos obtiveram ganhos na QV, após três meses de Pilates.

Um estudo envolvendo amostras mistas de homens e mulheres, demonstrou que o Pilates pode reduzir a dor e incapacidade, porém não encontrou diferenças entre os sexos. ${ }^{17} \mathrm{Da}$ mesma forma, a investigação da QV e atividade física realizada em 199 idosos brasileiros, (117 homens e 82 mulheres), também não identificou diferença na autopercepção da QV entre homens e muIheres. ${ }^{18}$ Este estudo apontou melhora na autopercepção da QV do sexo feminino, quando comparada com o masculino no mesmo período, divergindo das pesquisas apresentadas. Neste trabalho, a proposta foi investigar de forma global os participantes por sexo, não limitando a pesquisa somente a pacientes saudáveis ou com patologias específicas, bem como a autopercepção pode ser influenciada por ' $n$ ' motivos como o ambiente de prática, a formação do instrutor, a avaliação inicial da QV, fase da vida, entre outros. Analisando de forma mais abrangente o público feminino desta pesquisa por idade, apontam-se melhores ganhos na faixa etária de 41 a 50 anos. Tais fatores podem estar relacionados com o período do climatério, compreendido entre 40 e 55 anos, que na maioria das mulheres, desencadeia sintomas vasomotores, psicológicos, urogenitais, sexuais e de distúrbios do sono, derivados do declínio de estrogênio, ${ }^{19}$ além da menopausa, que provoca o hipoestrogenismo e também é um fator que pode contribuir para a flacidez da musculatura do assoalho pélvico, o que predispõe o surgimento de sintomas de perdas urinárias a pequenos e moderados esforços, além de predispor a perda a massa óssea e massa muscular global entre outros fatores. ${ }^{20}$ O Pilates parece melhorar a QVRS avaliada, utilizando SF-36 em mulheres no climatério ${ }^{21}$, pois atua sobre o assoalho pélvico, bem como pode atuar na fase idosa, trazendo efeitos benéficos sobre a dor capacidade funcional e qualidade de vida em pacientes com osteoporose pós-menopáusica, demonstrando ser uma alternativa segura e eficaz para estas pacientes.

A prática de atividade física (AF) adotada pelo Brasil é baseada na recomendação da Organização Mundial da Saúde (OMS), que, por sua vez, endossa as diretrizes estadunidenses. A última atualização da OMS, de 2010 , orienta adultos à prática de pelo menos 150 minutos por semana de AF moderada ou 75 minutos por semana de AF vigorosa, em sessões de pelo menos 10 minutos de duração, sem determinação de frequência semanal. ${ }^{22}$ Isto posto, considerando o tempo de duração de 50 minutos de uma sessão de Pilates, os resultados deste estudo conferem com as recomendações mundiais e demonstram que aqueles que praticaram com uma frequência de três vezes semanais obtiveram os melhores resultados. Assim sendo, esta investigação corrobora com Bize et al. ${ }^{23}$ que encontrou melhores taxas de QV em algumas dimensões nos indivíduos que praticam atividade física dentro dos níveis recomendados, do que nos indivíduos que não praticam, o que sugere que a atividade física pode influenciar os resultados em dimensões psicológicas, físicas e sociais. ${ }^{23}$

Contudo, quando se aborda o tema Pilates não há um consenso acerca da melhor frequência semanal. Outrossim, o tempo de intervenção varia desde quatro semanas, até um ano de duração e há poucas coincidências entre os autores relacionando a frequência semanal e o tempo de intervenção total, sugerindo que não existem protocolos estabelecidos a nível de pesquisa, para exercícios de Pilates que são comumente usados. ${ }^{21}$ As evidências indicam que, quanto mais próximo da prática regular de 150 minutos semanais recomendadas pela OMS, melhores os resultados.

$\mathrm{O}$ método tem se demonstrado eficiente na prevenção e no tratamento de várias patologias, além da literatura apontar como vantagens estimular a circulação e melhorar o condicionamento físico, que também ajudam na prevenção de lesões e proporcionam alívios das dores, com ótimos resultados. ${ }^{21,24}$ Nesta pesquisa foram encontrados 11 tipos de patologias, sendo seis doenças músculo-esqueléticas, uma vascular, uma gastrointestinal, uma genitourinária, uma óptica e outra do sistema neurológico. O método pode ser benéfico em doenças músculo-esqueléticas, como por exemplo, a dor lombar, ${ }^{23,25,26}$ corroborando com a presente pesquisa, com destaque para a maior evolução identificada na lombalgia sem causa aparente, bem como na hérnia discal e espondilite anquilosante lombar. Para Altan et al. ${ }^{25}$, que avaliaram a espondilite anquilosante, após um programa de pilates, constataram que pacientes com patologias instaladas, obtém maiores ganhos na QV, após a prática regular de três meses, confirmando os achados deste estudo. ${ }^{25}$ Natour et al. ${ }^{26}$ após pesquisarem a influência do Pilates em pacientes com dor lombar crônica, concluíram que o método foi eficaz na diminuição da dor, melhorou a função e a OV, ressaltando que é uma técnica segura para este tipo de pacientes, ${ }^{26}$ contrário à revisão sistemática e meta-análise de que concluiu não haver evidencias de que o método melhora a dor ou a funcionalidade destes pacientes, além de recomendar que deve ser cuidadosamente considerada para tais pacientes. ${ }^{27}$ Em outro estudo com pacientes de dor lombar crônica, divididos em dois grupos, um praticando o método pilates e o outro recebeu uma cartilha de exercícios "domésticos", foi observada uma pequena melhora nos resultados na dor, do grupo pilates, todavia que não foi estatisticamente significativa após 06 meses, ${ }^{28}$ resultados que condizem com este estudo que identificou um melhora global no domínio 
"dor" e demonstraram que os pacientes com patologias na coluna lombar obtiveram acréscimos em seus escores final da QV.

O método Pilates tem se mostrado benéfico não apenas em doenças musculoesqueléticas. Mulheres com endometriose, que se exercitam regularmente, tinham uma dimensão de saúde mental significativamente maior; ${ }^{23}$ tal fato sustenta os resultados encontrados na presente pesquisa, para esta patologia em questão, que apresentou evolução na saúde mental, bem como na QV total.

As pesquisas sugerem que a atividade física regular, como método de gestão de enxaqueca, pode acarretar num efeito benéfico sobre a frequência e intensidade, bem como sobre a duração dos ataques e bem-estar do paciente. Ainda assim, o conhecimento sobre dor de cabeça e atividade física é limitado e poucos estudos tem avaliado este relacionamento. ${ }^{29}$ Contudo, a maioria dos estudos não encontrou redução significativa da frequência ou duração das crises de cefaléia, ${ }^{30}$ mas sugere que o exercício ativa sinais de neurotransmissor endógeno, que poderiam ser eficazes na redução da intensidade da dor da enxaqueca. ${ }^{31} \mathrm{O}$ resultado positivo para a paciente com a patologia enxaqueca foi notável nesta pesquisa, corroborando com os estudos acerca dos benefícios da atividade física nestes casos.

Independente do sexo e idade, a prática da atividade física traz consigo uma melhora em todos os aspectos da qualidade de vida, ${ }^{3}$ equivalente aos efeitos percebidos neste estudo, visto que dos oito domínios que compõem o questionário, seis tiveram seus escores médios aumentados, um permaneceu sem alteração e apenas um, "limitação por aspectos emocionais", mostrou declínio na $\mathrm{QV}$, influenciado por demais fatores que não concernem aos exercícios de Pilates.

Este estudo apresentou limitações, no que se refere ao questionário SF36 que se destaca como sendo autoaplicável. No entanto, no decorrer deste inquérito, notou-se dificuldade na interpretação das questões, a não regularidade e a desistência da prática por parte dos pesquisados, foi outro fator, promovendo a exclusão na pesquisa.

\section{CONSIDERACִÕES FINAIS}

Após três meses de prática de Pilates, foi possível observar uma discreta evolução na QV dos praticantes. De forma estratificada, os acréscimos mais evidentes ocorreram, na faixa etária de 41 a 50 anos, para o sexo feminino, para frequência de três vezes por semana e em pacientes com patologia. Recomenda-se a continuidade da investigação, com uma amostra maior e considerando um tempo de intervenção superior a três meses.

\section{REFERÊNCIAS}

1. Gordia AP, Quadros TMB, Oliveira MTC, Campos W. Qualidade de vida: contexto histórico, definições, avaliação e fatores associados. Rev Bras Qualid Vida 2011;3(1):40-52. DOI: http://dx.doi.org/10.3895/S2175-08582011000100005.

2. Araújo DSMS, Araújo, CGS. Aptidão física, saúde e qualidade de vida relacionada à saúde em adultos. Rev Bras
Med Esporte 2000;6(5):194-203. DOI: 10.1590/S151786922000000500005.

3. Silva RS, Silva I, Silva RA, Souza L, Tomasi E. Atividade física e qualidade de vida. Rev. Ciência \& Saúde Coletiva 2010:15(1):115-20. DOI: 10.1590/S141381232010000100017

4. Ferreira C, Novaes G, Vianna J, Carneiro A, Menezes L. O método pilates sobre a resistência muscular localizada em mulheres adulta. Motricidade 2007;3(4):76-81.

5. Assumpção, LOT; Morais, PP.; Fontoura, H. Relação Entre Atividade Física, Saúde e Qualidade De Vida. Notas Introdutórias. EF Y Desp 2002;52:1-3 Disponível em: http:// www.efdeportes.com/efd52/saude.htm. Acesso em 15 de agosto de 2015.

6. Pilates JH. A obra completa de Joseph Pilates. Sua Saúde e $\mathrm{O}$ Retorno à Vida pela Contrologia. 1. Ed. Brasileira. São Paulo: Phorte Editora, 2010.

7. Rodrigues BGS, Cader CS, Torres NVOB, Oliveira EM, Dantas EHM. Autonomia funcional de idosas praticantes de pilates. Fisioterapia e Pesquisa 2010;17(4):300-05. DOI: 10.1590/ S1809-29502010000400003.

8. Araújo LM, Silva JMN, Bastos WT, Ventura PL. Diminuição da dor em mulheres com dismenorreia primaria, tratadas pelo método pilates. Rev Dor 2012;13(2):119-23. DOI: 10.1590/ S1806-00132012000200004

9. Ware JE, Sherbourne CD. The MOS 36-Item Short-Form Health Survey (SF-36): I. Conceptual framework and item selection. Med Care 1992;30:473-83.

10. Ciconelli RM. Tradução para o português e validação do questionário genérico de avaliação de qualidade de vida. 1997. 120p. Tese (doutorado em Ciências Médicas) - Escola Paulista de Medicina da Universidade Federal de São Paulo, São Paulo, 1997.

11. Pereira ÉF, Teixeira CS, Santos A. Qualidade de vida: abordagens, conceitos e avaliação. Revista Bras. Educ. Fis. Esporte 2012;26(2):241-50. DOI: 10.1590/S180755092012000200007.

12. Minayo MCS, Hartz, ZMA, Marchiori, P. Qualidade de vida e saúde: um debate necessário. Ciênc. Saúde Coletiva 2000;5(1):7-18. DOI: 10.1590/S1413-81232000000100002.

13. Guiselini M. Aptidão física saúde bem-estar: Fundamentos teóricos e exercícios práticos. 2.ed. São Paulo: Phorte; 2006.

14. Bezerra MJ, Perfeito RS. Variação do humor por meio de exercícios de pilates em adolescentes acautelados. Nova Fisio Digital 2013;16(90).

15. Takata, Y, Ansai, T, Soh, I, Awano, S, Yoshitake, Y, Kimura, et al. Quality of life and physical fitness in an 85-year-old population. Arch Gerontol Geriatr 2010;50(3):272-6. DOI: 10.1016/j.archger.2009.04.005.

16. Reis LA, Mascarenhas CHM, Lyra JE. Avaliação da qualidade de vida em idosos praticantes e não praticantes do método Pilates. C\&D-Revista Eletrônica da Fainor, Vitoria da Conquista 2011;4(1):38-51

17. Leopoldino AAO, Avelar NCP, Passos Jr GB, Santana Jr NAP, Texeira Jr VP, Lima VP, Vitorino D FM. Effect of Pilates on sleep quality and quality of life of sedentary population. European Journal of Integrative Medicine 2014;6(6):631-6. DOI: 10.1016/j.jbmt.2012.10.001.

18. Mazzarino M, Kerr D, Wajswelner H, Morris ME. Pilates method for women's health: systematic review of randomized controlled trials. Physical Medicine and Rehabilitation. 22 April, 2015. Disponível em: http://www.archives-pmr.org/ article/S0003-9993(15)00324-X/abstract. Acessado em 31 
agosto 2015.

19. Porto DB, Guedes DP, Fernandes ERA, Reichert FF. Perceived quality of life and physical activity in Brazilian older adults. Revista Motricidade 2012;8(1):33-41. DOI: 10.6063/ motricidade.8(1). 237.

20. Silva Filho EA, Costa AM. Avaliação da qualidade de vida de mulheres no climatério atendidas em hospital-escola na cidade do Recife. Rev Bras Ginecol Obstet 2008;30(3):11320. DOI: 10.1590/S0100-72032008005000001.

21. Baracho E. Fisioterapia aplicada à obstetrícia, urologia e aspectos de mastologia. 4. ed. Rio de Janeiro: Guanabara Koogan, 2007.

22. Fuentes GR, Oliveira IM, Berea HO, Gardamala MDO. An observational study on the effects of pilates on quality of life in women during menopause. European Journal of Integrative Medicine 2014;6(6):631-6. DOI: 10.1016/j. eujim.2014.08.003.

23. Lima POP, Gonçalvez PS. Percepção de saúde e qualidade de vida: um Inquérito com praticantes de pilates. Rev Fisioter $\mathrm{S}$ Fun., 2014;3(1):11-7.

24. Bize R, Johnson JA, Plotnikoff RC. Physical activity level and health-related quality of life in the general adult population: a systematic review. Prev Med 2007;45:401-15. DOI: 10.1016/j.ypmed.2007.07.017.

25. Borges J, Baptista A F, Santana N, Souza I, Kruschewsky RA, Galvão- Castro B, Sá KN. Pilates exercises improve low back pain and quality of life in patients with HTLV-1 virus: a randomized crossover clinical trial. Journal of Bodywork and movement therapies 2014;18(1):68-74. DOI: 10.1016/j. jbmt.2013.05.010.
26. Altan L, Korkmaz N, Dizdar M, Yurtkuran M. Effect of Pilates training on people with ankylosing spondylitis. Rheumatology International 2012;32(7):2093-99. DOI: 10.1007/s00296011-1932-9

27. Natour J, Cazotti LA, Ribeiro LH, Baptista AS, Jones A. Pilates improves pain, function and quality of life in patients with chronic low back pain: a randonized controlled trial. Clinical Rehabilitation 2015;29(1):59-68. DOI: 10.1177/0269215514538981.

28. Pereira LM, Obara K, Dias JM, Menacho MO, Guariglia DA, Schiavoni D, Pereira H M, Cardoso JR. Comparing the Pilates method with no exercise or lumbar stabilizartion for pain and functionality in pain: systemacic review and meta-analysis. Clinical Rehabilitation 2012;26(1):10-20. DOI: 10.1177/0269215511411113.

29. Miyamoto, GC, Costa LOP, Galvanin T, Cabral CMN. Efficacy of the additionof modified pilates exercices to a minimal intervention in patients with chronic low back pain: a randomized controlled trial. Phys Ther 2013;93(3):310-20. DOI: $10.2522 / p t j .20120190$.

30. Varkey E, Hagen K, Zwart JA. Phisical actvity and headache: results from the nord - trodelang health study. Cephalalgia 2008;28(12):1292-97. DOI: 10.1111/j.14682982.2008.01678.x.

31. Busch V, Gaul C. Exercise in migraine therapy-ls there any evidence for efficacy? A critical review. Headache 2008;48(6):890-9.

32. Ahn $\mathrm{AH}$. Why does increased exercise decrease migraine. Curr Pain Headache Rep 2013;17(12):319. DOI: 10.1007/ s11916-013-0379-y. 